\title{
DIVISION VI WORKING GROUPS
}

Division VI WG

Division VI WG

Division VI WG
Astrochemistry

Star Formation

Planetary Nebulae

\section{Working Group Business Meetings}

\subsection{Planetary Nebulae}

During the last business meeting of the WG on PN during the GA09, several topics were addressed. The renovation of the WG members was discussed. We agreed that members will be renewed during the PN IAU symposiums, and that the maximum duration of the term will be 10 years. It was decided that the next IAU PN Symp will be held in Puerto de la Cruz, Tenerife, in 2011. It seemed appropriate to hold the symposium in the Canary Islands in recognition of the new $10.4 \mathrm{~m}$ GTC telescope recently inaugurated on La Palma and its importance to the field of planetary nebulae.

The publication of links of interest in the field of PN on the PNWG webpage (http://www.iac.es/proyecto/PNgroup/) was also discussed.

\subsection{Organising Committee:}

A Manchado (Spain, Chair), M Barlow (UK), R Corradi (Spain), Y-H Chu (USA), S Deguchi (Japan), A Frank (USA), G Jacoby (USA), S Kwok (China), A López (Mexico), W Maciel (Brazil), R Méndez (USA), Q Parker (Australia), D Schoenberner (Germany), L Stanghellini (USA), A Zijlstra (UK) 